\title{
EVANS-KISHIMOTO TYPE ARGUMENT FOR ACTIONS OF DISCRETE AMENABLE GROUPS ON MCDUFF FACTORS
}

\author{
TOSHIHIKO MASUDA
}

\begin{abstract}
We apply the Evans-Kishimoto type argument to centrally free actions of discrete amenable groups on McDuff factors, and classify them. Especially, we present a different proof that the ConnesTakesaki modules are complete cocycle conjugacy invariants for centrally free actions of discrete amenable groups on injective factors.
\end{abstract}

\section{Introduction}

In the theory of operator algebras, the study of automorphism groups is one of the most important subjects. Especially, since Connes succeeded in classifying automorphisms of the approximately finite dimensional (AFD) factor of type $\mathrm{II}_{1}$ in [4] and [1], classification of actions of discrete amenable groups on injective factors has been solved in [9], [16], [19], [11] and finally in [10].

The strategy of Connes's classification is called the model action splitting argument. At first he constructed tensor product type model automorphisms (or actions) on the AFD type $\mathrm{II}_{1}$ factor. Then he showed "the model action splitting", i.e., every automorphism contains model automorphisms as tensor product components after an appropriate inner perturbation, and then proved that it is cocycle conjugate to the model automorphism. In his argument, the Rohlin property for automorphisms plays a crucial role. Namely, he showed the non-commutative version of a Rohlin type theorem for a certain class of automorphisms in [1]. By means of the Rohlin type theorem, he proved the stability (or 1-cohomology vanishing theorem) for automorphisms via a Shapiro type argument. Connes's argument has been developed by Jones for finite groups in [9], and by Ocneanu for general discrete amenable groups in [16]. Especially, the Rohlin type theorem was extended to the case of discrete amenable groups by Ocneanu, and he proved several cohomology vanishing theorems in [16].

Received May 23, 2005. 
On the other hand, another approach has been made in the study of automorphisms of $C^{*}$-algebras. In [6], Evans and Kishimoto developed the intertwining argument for classification of automorphisms with the Rohlin property. In their approach, they compare two given automorphisms directly without using model actions. Consequently they obtained classification results for a wide class of automorphisms. (In the $C^{*}$-algebra case, the model action splitting argument forces us to make a strict restriction on actions.) Their intertwining argument has been further developed in [15] for automorphisms of purely infinite simple $C^{*}$-algebras, and for finite group actions in [8].

In this paper, we apply the Evans-Kishimoto type intertwining argument to actions of discrete amenable groups on McDuff factors based on Ocneanu's Rohlin type theorem. Our main theorem says if two centrally free actions of a discrete amenable group on a McDuff factor differ up to approximately inner automorphisms, then they are cocycle conjugate. As a corollary, we get the complete classification of centrally free actions of discrete amenable groups on injective factors in terms of the Connes-Takesaki module by using the characterization of approximately inner automorphisms in [11]. Hence this is an another proof of the classical classification results in [1], [9], [16], [19], [11] for centrally free actions. However our approach seems to be more unified and simple, and this is an advantage of our theory.

Our result is also applicable to the classification of group actions on subfactors by a suitable modification. For example, we present a different proof of Popa's result in [17, Theorem 3.1]. (We remark that the classification result of strongly amenable subfactors of type $\mathrm{II}_{1}$ by Popa in [17] is crucial in our argument.)

AcKNowledgements. The author is grateful to Professor Izumi, Professor Katayama, and Professor Takesaki for comments on this work. He is supported by Grant-Aid for Scientific Research, Japan Society for the Promotion of Science.

\section{Preliminaries and notations}

Let $M$ be a von Neumann algebra. For $\varphi \in M_{*}^{+}$, we let $\|x\|_{\varphi}=\sqrt{\varphi\left(x^{*} x\right)}$, $\|x\|_{\varphi}^{\#}=\sqrt{\left(\|x\|_{\varphi}^{2}+\left\|x^{*}\right\|_{\varphi}^{2}\right) / 2},|x|_{\varphi}=\varphi(|x|)$. Note that $|x|_{\varphi}$ is not necessarily a norm unless $\varphi$ is tracial, since it is not subadditive. For $x \in M$ and $\varphi \in M_{*}$, $x \varphi, \varphi x \in M_{*}$ are defined as $x \varphi(y)=\varphi(y x)$ and $\varphi x(y)=\varphi(x y)$ respectively. We set $[x, \varphi]=x \varphi-\varphi x$. To avoid possible confusions, we often denote $x \varphi$ and $\varphi x$ by $x \cdot \varphi$ and $\varphi \cdot x$ respectively. We denote $\phi \circ \alpha^{-1}$ by $\alpha(\phi)$ for $\phi \in M_{*}$ and $\alpha \in \operatorname{Aut}(M)$.

We use the notation $A \Subset B$ when $A$ is a finite subset in $B$, and denote the cardinality of $A$ by $|A|$. 
Fix a free ultrafilter $\omega$ over N. We define $M_{\omega}$ and $M^{\omega}$ as in [16]. Each $\alpha \in \operatorname{Aut}(M)$ gives automorphisms $\alpha^{\omega} \in \operatorname{Aut}\left(M^{\omega}\right)$, and $\left.\alpha^{\omega}\right|_{M_{\omega}} \in \operatorname{Aut}\left(M_{\omega}\right)$. For $\varphi \in M_{*}$ and $X=\left(x_{n}\right) \in M^{\omega}, \varphi^{\omega}(X):=\lim _{n \rightarrow \omega} \varphi\left(x_{n}\right)$ is a normal functional on $M^{\omega}$, which we denote by $\varphi$ for simplicity. When $M$ is a factor, $\tau_{\omega}(X):=\lim _{n \rightarrow \omega} x_{n}(\in \mathrm{C})$ always exists in the $\sigma$-weak topology for $X=$ $\left(x_{n}\right) \in M_{\omega}$, and $\tau_{\omega}$ is a tracial state on $M_{\omega}$. We denote by $|X|_{1}$ the $L^{1}$-norm with respect to $\tau_{\omega}$.

We next collect some fundamental and useful inequalities in this paper.

Lemma 2.1. The following inequalities hold for $\varphi \in M_{*}^{+}, x \in M, x_{i} \in M^{\omega}$ and $y_{i} \in M_{\omega}$.

(1) $\|x \cdot \varphi\| \leq \sqrt{\|\varphi\|}\|x\|_{\varphi},\|\varphi \cdot x\| \leq \sqrt{\|\varphi\|}\left\|x^{*}\right\|_{\varphi},\|[x, \varphi]\| \leq 2 \sqrt{\|\varphi\|\|x\|_{\varphi}^{\#}}$.

(2) $\|x\|_{\varphi}^{2} \leq\|x \cdot \varphi\|\|x\|,\left\|x^{*}\right\|_{\varphi}^{2} \leq\|\varphi \cdot x\|\|x\|$.

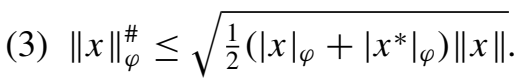

(4) $\left|\sum_{i} x_{i} y_{i}\right|_{\varphi} \leq \sum_{i}\left\|x_{i}\right\|\left|y_{i}\right|_{1}$.

Proof. It is elementary to see (1), (2), (3). See [16, Lemma 7.1] for the proof of (4).

Next we recall Ocneanu's Rohlin type theorem, which is a main tool in the proof of Lemma 3.4 below.

Theorem 2.2 ([16, Theorem 6.1]). Let $M$ be a McDuff factor, $G$ a discrete amenable group, and $\alpha$ an action of $G$ on $M_{\omega}$ which is strongly free and semiliftable. Let $\varepsilon>0$, and $\left\{K_{i}\right\}_{i \in I}$ be an $\varepsilon$-paving family. Then there exists a partition of unity $\left\{E_{i, k}\right\}_{i \in I, k \in K_{i}} \subset M_{\omega}$ such that

$$
\begin{gathered}
\sum_{i \in I}\left|K_{i}\right|^{-1} \sum_{k, l \in K_{i}}\left|\alpha_{k l^{-1}}\left(E_{i, l}\right)-E_{i, k}\right|_{1} \leq 5 \varepsilon^{\frac{1}{2}}, \\
{\left[\alpha_{g}\left(E_{i, k}\right), E_{j, l}\right]=0, \quad \text { for all } \quad g \in G, i, j \in I, k \in K_{i}, l \in K_{j} .}
\end{gathered}
$$

See [16] for the notation in the above theorem. Here we briefly explain how to construct an $\varepsilon$-paving family $\left\{K_{i}\right\}$. Fix $N \in \mathrm{N}$ such that $N>\frac{4}{\varepsilon} \log \varepsilon^{-1}$, and set $\delta:=(\varepsilon / 3)^{N}$. Let $K_{n+1}$ be a $\left(\delta\left|\bar{K}_{n}\right|^{-1}, \bar{K}_{n}\right)$-invariant finite set, where $\bar{K}_{n}:=\cup_{1 \leq i \leq n} K_{i}$. (In this paper, we say that $K$ is $(\varepsilon, F)$-invariant if $\mid K \cap$ $\bigcap_{g \in F} g^{-1} K|\geq(1-\varepsilon)| K \mid$.) Then $\left\{K_{i}\right\}_{1 \leq i \leq N}$ is shown to be an $\varepsilon$-paving family. In this construction, each $K_{i}$ can be chosen arbitrarily invariant. 


\section{Classification}

We state the main result in this paper.

THEOREM 3.1. Let $M$ be a McDuff factor, $G$ a countable discrete amenable group, and $\alpha, \beta$ centrally free actions of $G$ on $M$. If $\alpha_{g} \beta_{g}^{-1} \in \overline{\operatorname{Int}}(M)$ for every $g \in G$, then there exist an $\alpha$-cocycle $v_{g}$ and $\theta \in \overline{\operatorname{Int}}(M)$ such that Ad $v_{g} \alpha_{g}=\theta \circ \beta_{g} \circ \theta^{-1}$. Moreover we can choose $v_{g}$ close to 1, i.e., for any given $\varepsilon>0, F \Subset G$ and $\varphi \in M_{*}^{+}$, we can choose $v_{g}$ so that $\left\|v_{g}-1\right\|_{\varphi}^{\#}<\varepsilon$ for any $g \in F$.

The assumption $\alpha_{g} \beta_{g}^{-1} \in \overline{\operatorname{Int}}(M)$ implies $\alpha_{g}=\lim _{n \rightarrow \infty} \operatorname{Ad} u_{g}^{n} \beta$ for some unitaries $u_{g}^{n} \in M$. However $\operatorname{Ad} u_{g}^{n} \beta_{g}$ is not necessary an action of $G$. Hence we need to take $u_{g}^{n}$ as $\beta$-cocycles at first.

Proposition 3.2. Let $\alpha, \beta$ be as in Theorem 3.1. Then there exist $\beta$-cocycles $u_{g}^{n}, n=1,2, \ldots$, such that $\alpha_{g}=\lim _{n \rightarrow \omega} \operatorname{Ad} u_{g}^{n} \beta_{g}$.

Proof. Since $\alpha_{g} \beta_{g}^{-1} \in \overline{\operatorname{Int}}(M)$, there exist unitaries $u_{g}^{n}, g \in G, n=$ $1,2, \ldots$, such that $\alpha_{g}=\lim _{n \rightarrow \infty} \operatorname{Ad} u_{g}^{n} \beta_{g}$. Set $U_{g}:=\left(u_{g}^{n}\right) \in M^{\omega}$. Note that $\alpha_{g}^{\omega}=\operatorname{Ad} U_{g} \beta_{g}^{\omega}$ holds on $M\left(\subset M^{\omega}\right)$. Set $u(g, h)=U_{g} \beta_{g}^{\omega}\left(U_{h}\right) U_{g h}^{*}$. Then it is easy to verify that $u(g, h) \in M_{\omega}$ and $\left\{\left.\operatorname{Ad} U_{g} \beta_{g}^{\omega}\right|_{M_{\omega}}, u(g, h)\right\}$ is a cocycle action on $M_{\omega}$. Moreover Ad $U_{g} \beta_{g}^{\omega}$ is strongly free in the sense of [16, Definition 5.6] by [16, Lemma 5.7]. By Ocneanu's 2-cohomology vanishing theorem [16, Proposition 7.4], we get $c_{g} \in U\left(M_{\omega}\right)$ such that $c_{g} \operatorname{Ad} U_{g} \beta_{g}^{\omega}\left(c_{h}\right) u(g, h) c_{g h}^{*}=$ 1 , which yields $c_{g} U_{g} \beta_{g}^{\omega}\left(c_{h} U_{h}\right)=c_{g h} U_{g h}$. Hence $W_{g}:=c_{g} U_{g}$ becomes a $\beta^{\omega}$-cocycle. Let $c_{g}=\left(c_{g}^{n}\right)$ be a representing sequence consisting of unitaries. Since $\left(c_{g}^{n}\right)$ is a centralising sequence, Ad $c_{g}^{n}$ converges to id ${ }_{M}$. Hence $\alpha_{g}=$ $\lim _{n \rightarrow \omega}$ Ad $c_{g}^{n} u_{g}^{n} \beta_{g}$. Set $w_{g}^{n}=c_{g}^{n} u_{g}^{n}$ and $w_{n}^{\prime}(g, h):=w_{g}^{n} \beta_{g}\left(w_{h}^{n}\right) w_{g h}^{n *}$. Then (Ad $w_{g}^{n} \beta_{g}, w_{n}^{\prime}(g, h)$ ) is a cocycle action, and the cocycle identity $W_{g} \beta_{g}^{\omega}\left(W_{h}\right)=$ $W_{g h}$ yields $\lim _{n \rightarrow \omega} w_{n}^{\prime}(g, h)=1$ in the $\sigma$-strong* topology. By Ocneanu's 2cohomology vanishing theorem [16, Theorem 7.6], we have $d_{g}^{n} \in U(M)$ such that $d_{g}^{n} \mathrm{Ad} w_{g}^{n} \beta_{g}\left(d_{h}^{n}\right) w_{n}^{\prime}(g, h) d_{g h}^{n *}=1$ and $\lim _{n \rightarrow \omega} d_{g}^{n}=1$ in the $\sigma$-strong* topology. Then $d_{g}^{n} w_{g}^{n}$ is a $\beta_{g}$-cocycle and $\left(d_{g}^{n} w_{g}^{n}\right)=\left(w_{g}^{n}\right)$ in $M^{\omega}$. It is easy to see $\alpha_{g} \beta_{g}^{-1}=\lim _{n \rightarrow \omega}$ Ad $d_{g}^{n} w_{g}^{n}$.

We get the following corollary immediately.

Corollary 3.3. Let $\alpha, \beta$ be as in Theorem 3.1. Then for any $\varepsilon>0, \Phi \Subset M_{*}$ and $F \Subset G$, there exists a $\beta$-cocycle $u_{g}$ such that $\left\|\alpha_{g}(\phi)-\operatorname{Ad} u_{g} \beta_{g}(\phi)\right\|<\varepsilon$ for every $g \in F$ and $\phi \in \Phi$.

Next we show an approximate 1-cohomology vanishing theorem, which plays a central role in our argument. 
Lemma 3.4. Let $\alpha$ be a centrally free action of $G$ on $M$. For any $\varepsilon>0$, $F=F^{-1} \Subset G, \Phi^{+} \Subset M_{*}^{+}$, and $\Phi \Subset M_{*}$, there exist $\delta>0$ and $\Psi \Subset M_{*}$ with the following property; for any $\alpha$-cocycle $\left\{v_{g}\right\}$ with $\left\|\left[v_{g}, \psi\right]\right\|<\delta, g \in F$, $\psi \in \Psi$, we can find $w \in U(M)$ such that $\|[w, \varphi]\|<\varepsilon$ for every $\varphi \in \Phi$, and $\left\|v_{g} \alpha_{g}\left(w^{*}\right) w-1\right\|_{\phi}^{\#}<\varepsilon$ for every $g \in F$ and $\phi \in \Phi^{+}$. When $\Phi=\emptyset, \Psi=\emptyset$ is possible.

Proof. Since every $\phi \in M_{*}$ is decomposed as $\phi=\phi_{1}-\phi_{2}+i\left(\phi_{3}-\phi_{4}\right)$, $\phi_{i} \in M_{*}^{+}$, it suffices to show the lemma in the case $\Phi=\Phi^{+} \Subset M_{*}^{+}$. We may assume $0<\varepsilon<1$ and $\|\phi\| \leq 1, \phi \in \Phi^{+}$. Fix $\varepsilon^{\prime}>0$ with $\varepsilon^{\prime}<(\varepsilon / 8)^{4}$. Let $\left\{K_{i}\right\}_{i \in I}$ be an $\varepsilon^{\prime}$-paving family such that each $K_{i}$ is $\left(\varepsilon^{\prime}, F\right)$-invariant. We may assume that all $K_{i}$ are in a subgroup of $G$ generated by $F$. Define Length $(g):=$ $\min \left\{n \mid g=h_{1} h_{2} \cdots h_{n}, h_{i} \in F\right\}$, and set $L$ by

$$
L:=\max \left\{\operatorname{Length}(g) \mid g \in K_{i}, i \in I\right\} .
$$

Fix $\delta>0$ such that $\sum_{i}\left|K_{i}\right|(L+1) \delta<\varepsilon / 3$. Define $\Psi$ by

$$
\Psi:=\Phi^{+} \cup \bigcup_{\substack{1 \leq k \leq L-1, g_{i} \in F}} \alpha_{g_{1} g_{2} \ldots g_{k}}^{-1}\left(\Phi^{+}\right) .
$$

By Ocneanu's Rohlin type Theorem, there exists a partition of unity $\left\{E_{i, k}\right\}_{i \in I, k \in K_{i}} \subset M_{\omega}$ such that

$$
\begin{gathered}
\sum_{i \in I}\left|K_{i}\right|^{-1} \sum_{k, l \in K_{i}}\left|\alpha_{k l^{-1}}\left(E_{i, l}\right)-E_{i, k}\right|_{1}<5 \varepsilon^{\prime \frac{1}{2}}, \\
{\left[\alpha_{g}\left(E_{i, k}\right), E_{j, l}\right]=0 \quad \text { for all } \quad g \in G, i, j \in I, k \in K_{i}, l \in K_{j} .}
\end{gathered}
$$

Then by [16, Corollary 6.1], we have

$$
\sum_{i \in I} \sum_{k \in K_{i} \cap g^{-1} K_{i}}\left|\alpha_{g}\left(E_{i, k}\right)-E_{i, g k}\right|_{1} \leq 10 \varepsilon^{\prime \frac{1}{2}}
$$

and

$$
\sum_{i \in I} \sum_{k \in K_{i} \backslash g^{-1} K_{i}}\left|E_{i, k}\right|_{1} \leq \varepsilon^{\prime}+5 \varepsilon^{\prime \frac{1}{2}}
$$

for any $g \in F$.

We show that $\delta$ and $\Psi$ defined above are the desired ones. Let $v_{g}$ be an $\alpha$ cocycle with $\left\|\left[v_{g}, \psi\right]\right\|<\delta, g \in F, \psi \in \Psi$. Set $W:=\sum_{i, k} v_{k}^{*} E_{i, k}$. First we estimate $\left\|v_{g} \alpha_{g}\left(W^{*}\right) W-1\right\|_{\phi}^{\#}$ for $g \in F$ as in the proof of [16, Proposition 7.2]. To this end, we investigate $\left|v_{g} \alpha_{g}\left(W^{*}\right) W-1\right|_{\phi}$ and $\left|\left(v_{g} \alpha_{g}\left(W^{*}\right) W-1\right)^{*}\right|_{\phi}$, and then use Lemma 2.1 . 
We divide

$$
v_{g} \alpha_{g}\left(W^{*}\right) W-1=\sum_{i \in I, k \in K_{i}} \sum_{j \in I, l \in K_{j}}\left(v_{g} \alpha_{g}\left(v_{k}\right) v_{l}^{*}-1\right) \alpha_{g}\left(E_{i, k}\right) E_{j, l}
$$

into three parts as follows.

$$
\begin{aligned}
\sum_{i \in I, k \in K_{i}} & \sum_{j \in I, l \in K_{j}}(*) \\
= & \sum_{j \in I, l \in K_{j}} \sum_{i, k \in K_{i} \cap g^{-1} K_{i}}(*)+\sum_{j \in I, l \in K_{j}} \sum_{j \in I, k \in K_{i} \backslash g^{-1} K_{i}}(*) \\
= & \sum_{j \in I, l \in K_{j}} \sum_{\substack{j \neq i \in I^{\prime}, k \in K_{i} \cap g^{-1} K_{i}}}(*)+\sum_{\substack{j \in I, l \in K_{j}, k \in K_{j} \cap g^{-1} K_{j}}}(*)+\sum_{j \in I, l \in K_{j}} \sum_{i, k \in K_{i} \backslash g^{-1} K_{i}}(*) \\
= & \sum_{\substack{j \in I, l \in K_{j}, k \in K_{j} \cap g^{-1} K_{j}, g k=l}}(*)+\sum_{\substack{j \in I, l \in K_{j}, k \in K_{j} \cap g^{-1} K_{j}, g k \neq l}}(*) \\
& +\sum_{j \in I, l \in K_{j}} \sum_{\substack{j \neq i \in I, k \in K_{i} \cap g^{-1} K_{i}}}(*)+\sum_{j \in I, l \in K_{j}} \sum_{i, k \in K_{i} \backslash g^{-1} K_{i}}(*) \\
= & \sum_{1}(*)+\sum_{2}(*)+\sum_{3}(*) .
\end{aligned}
$$

In $\sum_{1}$ we sum for $i=j, k \in K_{i} \cap g^{-1} K_{i}, g k=l$, in $\sum_{2}$ we sum for $i=j, k \in K_{i} \cap g^{-1} K_{i}, g k \neq l$, or $i \neq j, k \in K_{i} \cap g^{-1} K_{i}, l \in K_{j}$, and in $\sum_{3}$ we sum for $j \in I, l \in K_{j}, k \in K_{i} \backslash g^{-1} K_{i}$. Due to the cocycle identity, we have $\sum_{1}\left(v_{g} \alpha_{g}\left(v_{k}\right) v_{l}^{*}-1\right) \alpha_{g}\left(E_{i, k}\right) E_{j, l}=\sum_{j}\left(v_{g} \alpha_{g}\left(v_{k}\right) v_{g k}^{*}-1\right) \alpha_{g}\left(E_{i, k}\right) E_{j, g k}=0$, and $\sum_{1}$ part vanishes. Hence

$$
\begin{aligned}
& \left|v_{g} \alpha_{g}\left(W^{*}\right) W-1\right|_{\phi} \\
& \quad=\left|\sum_{2}\left(v_{g} \alpha_{g}\left(v_{k}\right) v_{l}^{*}-1\right) \alpha_{g}\left(E_{i, k}\right) E_{j, l}+\sum_{3}\left(v_{g} \alpha_{g}\left(v_{k}\right) v_{l}^{*}-1\right) \alpha_{g}\left(E_{i, k}\right) E_{j, l}\right|_{\phi} \\
& \quad \leq 2 \sum_{2}\left|\alpha_{g}\left(E_{i, k}\right) E_{j, l}\right|_{1}+2 \sum_{3}\left|\alpha_{g}\left(E_{i, k}\right) E_{j, l}\right|_{1}
\end{aligned}
$$

holds by Lemma 2.1(4). Similarly we have

$$
\left|\left(v_{g} \alpha_{g}\left(W^{*}\right) W-1\right)^{*}\right|_{\phi} \leq 2 \sum_{2}\left|\alpha_{g}\left(E_{i, k}\right) E_{j, l}\right|_{1}+2 \sum_{3}\left|\alpha_{g}\left(E_{i, k}\right) E_{j, l}\right|_{1} .
$$


We estimate the $\sum_{2}$ part. Then

$$
\begin{aligned}
& \sum_{2}\left|\alpha_{g}\left(E_{i, k}\right) E_{j, l}\right|_{1} \\
& =\sum_{\substack{j \in I, l \in K_{j}}} \sum_{\substack{i \neq j, k \in K_{i} \cap g^{-1} K_{i}}}\left|\alpha_{g}\left(E_{i, k}\right) E_{j, l}\right|_{1}+\sum_{\substack{i \in I, l \in K_{i}, k \in K_{i} \cap g^{-1} K_{i}, g k \neq l}}\left|\alpha_{g}\left(E_{i, k}\right) E_{i, l}\right|_{1} \\
& =\sum_{\substack{i \in I, k \in K_{i} \cap g^{-1} K_{i}}}\left|\alpha\left(E_{i, k}\right)\left(1-\sum_{l} E_{i, l}\right)\right|_{1}+\sum_{\substack{i \in I, k \in K_{i} \cap g^{-1} K_{i}}}\left|\alpha_{g}\left(E_{i, k}\right) \sum_{l \in K_{i}, l \neq g k} E_{i, l}\right|_{1} \\
& =\sum_{i \in I, k \in K_{i} \cap g^{-1} K_{i}}\left|\alpha_{g}\left(E_{i, k}\right)\left(1-E_{i, g k}\right)\right|_{1} \\
& =\sum_{i \in I, k \in K_{i} \cap g^{-1} K_{i}}\left|\left(\alpha_{g}\left(E_{i, k}\right)-E_{i, g k}\right)\left(1-E_{i, g k}\right)\right|_{1} \\
& \leq \sum_{i \in I, k \in K_{i} \cap g^{-1} K_{i}}\left|\alpha_{g}\left(E_{i, k}\right)-E_{i, g k}\right|_{1} \\
& \leq 10 \varepsilon^{\prime \frac{1}{2}}
\end{aligned}
$$

holds.

The estimate of the $\sum_{3}$ part is given as follows.

$$
\begin{aligned}
\sum_{3}\left|\alpha_{g}\left(E_{i, k}\right) E_{j, l}\right|_{1} & =\sum_{j \in I, l \in K_{j}} \sum_{\substack{i \in I, k \in K_{i} \backslash g^{-1} K_{i}}}\left|\alpha_{g}\left(E_{i, k}\right) E_{j, l}\right|_{1}=\sum_{\substack{i \in I, k \in K_{i} \backslash g^{-1} K_{i}}}\left|E_{i, k}\right|_{1} \\
& \leq \varepsilon^{\prime}+5 \varepsilon^{\prime \frac{1}{2}} \leq 6 \varepsilon^{\prime \frac{1}{2}} .
\end{aligned}
$$

Summing up, we get $\left|v_{g} \alpha_{g}\left(W^{*}\right) W-1\right|_{\phi} \leq 32 \varepsilon^{\prime \frac{1}{2}}$ and $\mid\left(v_{g} \alpha\left(W^{*}\right) W-\right.$ $1)\left.^{*}\right|_{\phi} \leq 32 \varepsilon^{\prime \frac{1}{2}}$. By Lemma 2.1(3), we have

$$
\left\|v_{g} \alpha_{g}\left(W^{*}\right) W-1\right\|_{\phi}^{\#} \leq 8 \varepsilon^{\frac{1}{4}}<\varepsilon .
$$

We choose representing sequences $W=\left(w_{n}\right)$ consisting of unitaries, and $E_{i, k}=\left(e_{i, k}^{n}\right)$ such that $\left\{e_{i, k}^{n}\right\}_{i \in I, k \in K_{i}}$ is a partition of unity for each $n$. Set $a_{n}:=$ $\sum_{i, k} v_{k}^{*} e_{i, k}^{n}$. (Note that $a_{n}$ is not necessary a unitary.) Since $W=\left(w_{n}\right)=\left(a_{n}\right)$ in $M^{\omega},\left\{w_{n}-a_{n}\right\}$ converges to $0 \sigma$-strongly*. Choose a sufficiently large $n$ such that

$$
\begin{aligned}
\left\|v_{g} \alpha_{g}\left(w_{n}^{*}\right) w_{n}-1\right\|_{\phi}^{\#}<\varepsilon, & g \in F, \phi \in \Phi^{+}, \\
\left\|\left[\phi, e_{i, k}^{n}\right]\right\|<\delta, & \phi \in \Phi^{+}, i \in I, k \in K_{i}, \\
\left\|w_{n}-a_{n}\right\|_{\phi}^{\#}<\varepsilon / 3, & \phi \in \Phi^{+} .
\end{aligned}
$$


Set $w:=w_{n}, a:=a_{n}, e_{i, k}:=e_{i, k}^{n}$. (Note that we never use the assumption $\left\|\left[v_{g}, \psi\right]\right\|<\delta, \psi \in \Psi$, in the estimation of $\left\|v_{g} \alpha_{g}\left(w^{*}\right) w-1\right\|_{\phi}^{\#}$.) Next we show $\|[a, \varphi]\|<\varepsilon / 3, \varphi \in \Phi^{+}$. To show this, we estimate $\left\|\left[v_{g}, \varphi\right]\right\|, g \in K_{i}$, $\varphi \in \Phi^{+}$at first. We express $g$ as $g=g_{1} g_{2} \ldots g_{k}, g_{i} \in F, k \leq L$. Then it is easy to show $\left\|\left[v_{g}, \varphi\right]\right\| \leq \sum_{i=1}^{k}\left\|\left[v_{g_{i}}, \alpha_{g_{1} \ldots g_{i-1}}^{-1}(\varphi)\right]\right\|$. (When $i=1, \alpha_{g_{1} \ldots g_{i-1}}(\varphi)$ means $\varphi$.) Since each $\alpha_{g_{1} \ldots g_{i}}^{-1}(\varphi)$ is in $\Psi,\left\|\left[v_{g_{i}}, \alpha_{g_{1} \ldots g_{i-1}}^{-1}(\varphi)\right]\right\|<\delta$ follows by the assumption on $v_{g}$. Hence we have $\left\|\left[v_{g}, \varphi\right]\right\| \leq L \delta$. Finally,

$$
\begin{aligned}
\|[a, \varphi]\| & \leq \sum_{i \in I, k \in K_{i}}\left\|\left[v_{k}^{*} e_{i, k}, \varphi\right]\right\| \leq \sum_{i \in I, k \in K_{i}}\left\|\left[v_{k}^{*}, \varphi\right] e_{i, k}\right\|+\left\|v_{k}^{*}\left[e_{i, k}, \varphi\right]\right\| \\
& \leq \sum_{i \in I, k \in K_{i}}(L+1) \delta=\sum_{i \in I}\left|K_{i}\right|(L+1) \delta<\varepsilon / 3
\end{aligned}
$$

holds for $\varphi \in \Phi^{+}$.

By Lemma 2.1(1),

$$
\|[w, \varphi]\| \leq\|[a, \varphi]\|+\|[w-a, \varphi]\|<\varepsilon / 3+2\|w-a\|_{\varphi}^{\#}<\varepsilon
$$

holds for $\varphi \in \Phi^{+}$, and $w$ is a desired unitary.

REMARK. If we replace $v_{g} \alpha_{g}\left(W^{*}\right) W$ with $W v_{g} \alpha_{g}\left(W^{*}\right)$ in the above proof, we then conclude $\left\|w v_{g} \alpha_{g}\left(w^{*}\right)-1\right\|_{\phi}^{\#}<\varepsilon$.

Now we present a proof of Theorem 3.1 by means of Lemma 3.4.

Proof of Theorem 3.1. Fix a faithful normal state $\varphi_{0}$. Let $\Phi=\left\{\varphi_{i}\right\}_{i=0}^{\infty}$ be a countable dense subset in $M_{*}$, and set $\Phi_{n}:=\left\{\varphi_{i}\right\}_{i=0}^{n}$. Fix $G_{n} \Subset G$ such that $G_{n} \subset G_{n+1}, G_{n}^{-1}=G_{n}$ and $\bigcup_{n} G_{n}=G$.

We construct $w_{n}, v_{g}^{n}, \bar{v}_{g}^{n} \in U(M), \Phi_{n}^{\prime}, \Psi_{n} \Subset M_{*}, \Phi_{n}^{+} \Subset M_{*}^{+}, \delta_{n}>0$, and actions $\alpha_{g}^{(2 n)}, \beta_{g}^{(2 n-1)}$ of $G$ satisfying the below conditions inductively. (We set $\alpha^{(0)}:=\alpha, \beta^{(-1)}:=\beta$.)

$$
\left\|\beta_{g}^{(2 n-1)}(\varphi)-\alpha_{g}^{(2 n)}(\varphi)\right\|<1 / 2^{2 n}, \quad g \in G_{2 n}, \varphi \in \Phi_{2 n}^{\prime},(n \geq 1) .
$$

$(1.2 n+1) \quad\left\|\alpha_{g}^{(2 n)}(\varphi)-\beta_{g}^{(2 n+1)}(\varphi)\right\|<1 / 2^{2 n+1}$,

$$
g \in G_{2 n+1}, \varphi \in \Phi_{2 n+1}^{\prime},(n \geq 0) .
$$

$$
\begin{aligned}
& \left\|\beta_{g}^{(2 n-1)}(\psi)-\alpha_{g}^{(2 n)}(\psi)\right\|<\frac{\delta_{2 n-1}}{2}, \\
& \quad g \in G_{2 n-1}, \psi \in \bigcup_{g \in G_{2 n-1}} \beta_{g^{-1}}^{(2 n-1)}\left(\Psi_{2 n-1}\right),(n \geq 1) .
\end{aligned}
$$


$(2.2 n+1) \quad\left\|\alpha_{g}^{(2 n)}(\psi)-\beta_{g}^{(2 n+1)}(\psi)\right\|<\frac{\delta_{2 n}}{2}$,

$$
g \in G_{2 n}, \psi \in \bigcup_{g \in G_{2 n}} \alpha_{g^{-1}}^{(2 n)}\left(\Psi_{2 n}\right),(n \geq 1) .
$$

(3.n) $\left\|v_{g}^{n}-1\right\|_{\phi}^{\#}<1 / 4^{n}$,

$$
g \in G_{n-2}, \phi \in \Phi_{n-2}^{+},\left(G_{-1}=G_{0}=G_{1}, \Phi_{-1}^{+}=\Phi_{0}^{+}=\left\{\varphi_{0}\right\}\right),
$$

(4.n) $\left\|\left[w_{n}, \varphi\right]\right\|<1 / 4^{n}, \quad \varphi \in \Phi_{n-1}^{\prime},(n \geq 3)$.

$\bar{v}_{g}^{n}:=v_{g}^{n} \operatorname{Ad} w_{n}\left(\bar{v}_{g}^{n-2}\right), \quad\left(\bar{v}_{g}^{1}=v_{g}^{1}, \bar{v}_{g}^{2}=v_{g}^{2}\right)$,

$\alpha_{g}^{(2 n)}:=\operatorname{Ad} v_{g}^{2 n} \circ \operatorname{Ad} w_{2 n}^{*} \circ \alpha_{g}^{(2 n-2)} \circ \operatorname{Ad} w_{2 n}$,

$\beta_{g}^{(2 n-1)}:=\operatorname{Ad} v_{g}^{2 n-1} \circ \operatorname{Ad} w_{2 n-1}^{*} \circ \beta_{g}^{(2 n-3)} \circ \operatorname{Ad} w_{2 n-1}$,

$\Phi_{2 n}^{\prime}:=\Phi_{2 n} \cup \operatorname{Ad} w_{2 n-1}^{*} w_{2 n-3}^{*} \cdots w_{1}^{*}\left(\Phi_{2 n}\right) \cup\left\{\bar{v}_{g}^{2 n-1} \varphi_{0}, \varphi_{0} \bar{v}_{g}^{2 n-1}\right\}_{g \in G_{2 n-1}}$,

$\Phi_{2 n+1}^{\prime}:=\Phi_{2 n+1} \cup \operatorname{Ad} w_{2 n}^{*} w_{2 n-2}^{*} \cdots w_{2}^{*}\left(\Phi_{2 n+1}\right) \cup\left\{\bar{v}_{g}^{2 n} \varphi_{0}, \varphi_{0} \bar{v}_{g}^{2 n}\right\}_{g \in G_{2 n}},(n \geq 1)$

$\Phi_{n}^{+}:=\left\{\operatorname{Ad} \bar{v}_{g}^{n}\left(\varphi_{0}\right) \mid g \in G_{n}\right\}$.

Here $\delta_{2 n}$ and $\Psi_{2 n}\left(\delta_{2 n-1}\right.$ and $\left.\Psi_{2 n-1}\right)$ are chosen as in Lemma 3.4 for $\alpha^{(2 n)}$, $1 / 4^{2 n+2}, G_{2 n}, \Phi_{2 n}^{+}$and $\Phi_{2 n+1}^{\prime}\left(\right.$ resp. for $\beta^{(2 n-1)}, 1 / 4^{2 n+1}, G_{2 n-1}, \Phi_{2 n-1}^{+}$, and $\left.\Phi_{2 n}^{\prime}\right)$.

At first set $\Phi_{1}^{\prime}:=\Phi_{1}$ and fix a $\beta^{(-1)}$-cocycle $u_{g}^{1}$ such that

$$
\left\|\alpha_{g}^{(0)}(\varphi)-\operatorname{Ad} u_{g}^{1} \beta_{g}^{(-1)}(\varphi)\right\|<1 / 2, g \in G_{1}, \varphi \in \Phi_{1}^{\prime} .
$$

By Lemma 3.4, we get a unitary $w_{1}$ such that $\left\|u_{g} \beta_{g}^{(-1)}\left(w_{1}^{*}\right) w_{1}-1\right\|_{\varphi_{0}}^{\#}<1 / 4$, $g \in G_{1}$. Set $v_{g}^{1}:=u_{g}^{1} \beta_{g}^{(-1)}\left(w_{1}^{*}\right) w_{1}$, and $\beta_{g}^{(1)}:=\operatorname{Ad} u_{g}^{1} \circ \beta_{g}^{(-1)}=\operatorname{Ad} v_{g}^{1} \circ$ $\operatorname{Ad} w_{1}^{*} \circ \beta_{g}^{(-1)} \circ \operatorname{Ad} w_{1}$. Then we have

$$
\left\|\alpha_{g}^{(0)}(\varphi)-\beta_{g}^{(1)}(\varphi)\right\|<1 / 2
$$

and

$$
\left\|v_{g}^{1}-1\right\|_{\varphi_{0}}^{\#}<1 / 4
$$

for $g \in G_{1}$. Set $\bar{v}_{g}^{1}:=v_{g}^{1}, \Phi_{2}^{\prime}:=\Phi_{2} \cup \operatorname{Ad} w_{1}^{*}\left(\Phi_{2}\right) \cup\left\{\bar{v}_{g}^{1} \varphi_{0}, \varphi_{0} \bar{v}_{g}^{1}\right\}$, and $\Phi_{1}^{+}:=\left\{\operatorname{Ad} \bar{v}_{g}^{1}\left(\varphi_{0}\right)\right\}_{g \in G_{1}}$. By Lemma 3.4, we choose $\Psi_{1}$ and $\delta_{1}$ for $\beta_{g}^{(1)}, 1 / 4^{3}$, $G_{1}, \Phi_{1}^{+}$and $\Phi_{2}^{\prime}$, and the first step is finished. 
Next we take an $\alpha^{(0)}$-cocycle $u_{g}^{2}$ such that

(a.2) $\left\|\beta_{g}^{(1)}(\varphi)-\operatorname{Ad} u_{g}^{2} \alpha_{g}^{(0)}(\varphi)\right\|<\frac{1}{2^{2}}, \quad g \in G_{2}, \varphi \in \Phi_{2}^{\prime}$,

(b.2) $\left\|\beta_{g}^{(1)}(\psi)-\operatorname{Ad} u_{g}^{2} \alpha_{g}^{(0)}(\psi)\right\|<\frac{\delta_{1}}{2}, \quad g \in G_{1}, \psi \in \bigcup_{g \in G_{1}} \beta_{g^{-1}}^{(1)}\left(\Psi_{1}\right)$.

By Lemma 3.4, we get $w_{2} \in U(M)$ such that $\left\|u_{g}^{2} \alpha_{g}^{(0)}\left(w_{2}^{*}\right) w_{2}-1\right\|_{\varphi_{0}}^{\#}<1 / 4^{2}$ for $g \in G_{2}$. Set $v_{g}^{2}=\bar{v}_{g}^{2}:=u_{g}^{2} \alpha_{g}^{(0)}\left(w_{2}^{*}\right) w_{2}$ and $\alpha_{g}^{(2)}:=\operatorname{Ad} u_{g}^{2} \alpha_{g}^{(0)}=\operatorname{Ad} v_{g}^{2} \circ$ Ad $w_{2}^{*} \circ \alpha_{g}^{(0)} \circ$ Ad $w_{2}$. Then we get

$$
\left\|v_{g}^{2}-1\right\|_{\varphi_{0}}^{\#}<\frac{1}{4^{2}}, \quad g \in G_{2}
$$

By (a.2) and (b.2),

$$
\begin{array}{ll}
\left\|\beta_{g}^{(1)}(\phi)-\alpha_{g}^{(2)}(\phi)\right\|<\frac{1}{2^{2}}, & g \in G_{2}, \varphi \in \Phi_{2}^{\prime}, \\
\left\|\beta_{g}^{(1)}(\psi)-\alpha_{g}^{(2)}(\psi)\right\|<\frac{\delta_{1}}{2}, & g \in G_{1}, \psi \in \bigcup_{g \in G_{1}} \beta_{g^{-1}}^{(1)}\left(\Psi_{1}\right) .
\end{array}
$$

Set $\Phi_{3}^{\prime}:=\Phi_{3} \cup \operatorname{Ad} w_{2}^{*}\left(\Phi_{3}\right) \cup\left\{\bar{v}_{g}^{2} \varphi_{0}, \varphi_{0} \bar{v}_{g}^{2}\right\}_{g \in G_{2}}$ and $\Phi_{2}^{+}:=\left\{\operatorname{Ad} \bar{v}_{g}^{2} \varphi_{0} \mid g \in\right.$ $\left.G_{2}\right\}$. By Lemma 3.4, we choose $\delta_{2}$ and $\Psi_{2}$ for $\alpha^{(2)}, G_{2}, \Phi_{3}^{\prime}, \Phi_{2}^{+}$and $1 / 4^{4}$, and the second step is finished.

Suppose that we have constructed $\alpha_{g}^{(2 n)}, \beta_{g}^{(2 n-1)}, w_{2 n}, v_{g}^{2 n}, \bar{v}_{g}^{2 n}, \Phi_{2 n+1}^{\prime}, \delta_{2 n}$ and $\Psi_{2 n}$.

We choose a $\beta^{(2 n-1)}$-cocycle $u_{g}^{2 n+1} \in U(M)$ such that

$$
\begin{gathered}
(a .2 n+1) \quad\left\|\alpha_{g}^{(2 n)}(\varphi)-\operatorname{Ad} u_{g}^{2 n+1} \beta_{g}^{(2 n-1)}(\varphi)\right\|<\frac{1}{2^{2 n+1}}, \\
g \in G_{2 n+1}, \varphi \in \Phi_{2 n+1}^{\prime}, \\
(b .2 n+1) \quad\left\|\alpha_{g}^{(2 n)}(\psi)-\operatorname{Ad} u_{g}^{2 n+1} \beta_{g}^{(2 n-1)}(\psi)\right\|<\frac{\delta_{2 n}}{2}, \\
g \in G_{2 n}, \psi \in \bigcup_{g \in G_{2 n}} \alpha_{g^{-1}}^{(2 n)}\left(\Psi_{2 n}\right), \\
(c .2 n+1) \quad\left\|\alpha_{g}^{(2 n)}(\psi)-\operatorname{Ad} u_{g}^{2 n+1} \beta_{g}^{(2 n-1)}(\psi)\right\|<\frac{\delta_{2 n-1}}{2}, \\
g\left(G_{2 n-1}, \psi \in \bigcup_{g \in G_{2 n-1}} \beta_{g^{-1}}^{(2 n-1)}\left(\Psi_{2 n-1}\right) .\right.
\end{gathered}
$$


Then by $(2.2 n)$ and $(c .2 n+1)$ we get $\left\|\beta_{g}^{(2 n-1)}(\psi)-\operatorname{Ad} u_{g}^{2 n+1} \beta_{g}^{(2 n-1)}(\psi)\right\|<\delta_{2 n-1}$, $g \in G_{2 n-1}, \psi \in \beta_{g^{-1}}^{(2 n-1)}\left(\Psi_{2 n-1}\right)$,

which yields $\left\|\left[u_{g}^{2 n+1}, \psi\right]\right\|<\delta_{2 n-1}, g \in G_{2 n-1}, \psi \in \Psi_{2 n-1}$. By the choice of $\delta_{2 n-1}$ and $\Psi_{2 n-1}$, there exists a unitary $w_{2 n+1}$ such that

$$
\left\|u_{g}^{2 n+1} \beta_{g}^{(2 n-1)}\left(w_{2 n+1}^{*}\right) w_{2 n+1}-1\right\|_{\phi}^{\#}<1 / 4^{2 n+1}, \quad g \in G_{2 n-1}, \phi \in \Phi_{2 n-1}^{+},
$$

and

$$
\left\|\left[w_{2 n+1}, \varphi\right]\right\|<1 / 4^{2 n+1}, \quad \varphi \in \Phi_{2 n}^{\prime} .
$$

Set $v_{g}^{2 n+1}:=u_{g}^{2 n+1} \beta_{g}^{(2 n-1)}\left(w_{2 n+1}^{*}\right) w_{2 n+1}$ and $\beta_{g}^{(2 n+1)}:=\operatorname{Ad} u_{g}^{(2 n+1)} \beta_{g}^{(2 n-1)}=$ $\operatorname{Ad} v_{g}^{(2 n+1)} \circ \operatorname{Ad} w_{2 n+1}^{*} \circ \beta_{g}^{(2 n-1)} \circ \operatorname{Ad} w_{2 n+1}$. Then

$$
(3.2 n+1) \quad\left\|v_{g}^{2 n+1}-1\right\|_{\phi}^{\#}<\frac{1}{4^{2 n+1}}, \quad g \in G_{2 n-1}, \phi \in \Phi_{2 n-1}^{+} .
$$

By $(a .2 n+1)$ and $(b .2 n+1)$, we get

$(1.2 n+1) \quad\left\|\alpha_{g}^{(2 n)}(\varphi)-\beta_{g}^{(2 n+1)}(\varphi)\right\|<\frac{1}{2^{2 n+1}}$,

$$
g \in G_{2 n+1}, \varphi \in \Phi_{2 n+1}^{\prime} .
$$

$(2.2 n+1) \quad\left\|\alpha_{g}^{(2 n)}(\psi)-\beta_{g}^{(2 n+1)}(\psi)\right\|<\frac{\delta_{2 n}}{2}$,

$$
g \in G_{2 n}, \psi \in \bigcup_{g \in G_{2 n}} \alpha_{g^{-1}}^{(2 n)}\left(\Psi_{2 n}\right) .
$$

Set $\bar{v}_{g}^{2 n+1}:=v_{g}^{2 n+1} \operatorname{Ad} w_{2 n+1}^{*}\left(\bar{v}_{g}^{2 n-1}\right)$, and define

$$
\begin{aligned}
& \Phi_{2 n+2}^{\prime}:=\Phi_{2 n+2} \cup \operatorname{Ad} w_{2 n+1}^{*} w_{2 n-1}^{*} \cdots w_{1}^{*}\left(\Phi_{2 n+2}\right) \\
& \left.\Phi_{2 n+1}^{+}:=\left\{\overline{v d}_{g}^{2 n+1} \bar{v}_{g}^{2 n+1} \varphi_{0} \mid g \in \varphi_{2 n+1}\right\} . \varphi_{0} \bar{v}_{g}^{2 n+1}\right\}_{g \in G_{2 n+1}},
\end{aligned}
$$

By Lemma 3.4, we choose $\delta_{2 n+1}>0$ and $\Psi_{2 n+1} \Subset M_{*}$ for $\beta^{(2 n+1)}, 1 / 4^{2 n+3}$, $G_{2 n+1}, \Phi_{2 n+1}^{+}$and $\Phi_{2 n+2}^{\prime}$, and the $(2 n+1)$-st step is finished.

Next we choose an $\alpha^{(2 n)}$-cocycle $u_{g}^{2 n+2}$ such that

$(a .2 n+2) \quad\left\|\beta_{g}^{(2 n+1)}(\varphi)-\operatorname{Ad} u_{g}^{2 n+2} \alpha_{g}^{(2 n)}(\varphi)\right\|<\frac{1}{2^{2 n}}, g \in G_{2 n+2}, \varphi \in \Phi_{2 n+2}^{\prime}$, 


$$
\begin{gathered}
(b .2 n+2) \quad\left\|\beta_{g}^{(2 n+1)}(\psi)-\operatorname{Ad} u_{g}^{2 n+2} \alpha_{g}^{(2 n)}(\psi)\right\|<\frac{\delta_{2 n+1}}{2}, \\
g \in G_{2 n+1}, \psi \in \bigcup_{g \in G_{2 n+1}} \beta_{g^{-1}}^{(2 n+1)}\left(\Psi_{2 n+1}\right), \\
(c .2 n+2) \quad\left\|\beta_{g}^{(2 n+1)}(\psi)-\operatorname{Ad} u_{g}^{2 n+2} \alpha_{g}^{(2 n)}(\psi)\right\|<\frac{\delta_{2 n}}{2}, \quad g \in G_{2 n}, \psi \in \bigcup_{g \in G_{2 n}} \alpha_{g^{-1}}^{(2 n)}\left(\Psi_{2 n}\right) .
\end{gathered}
$$

By $(c .2 n+2)$ and $(2.2 n+1)$, we get

$$
\left\|\alpha_{g}^{(2 n)}(\psi)-\operatorname{Ad} u_{g}^{2 n+2} \alpha_{g}^{(2 n)}(\psi)\right\|<\delta_{2 n}, \quad g \in G_{2 n}, \psi \in \bigcup_{g \in G_{2 n}} \alpha_{g^{-1}}^{(2 n)}\left(\Psi_{2 n}\right)
$$

We thus have $\left\|\left[u_{g}^{2 n+2}, \psi\right]\right\|<\delta_{2 n}$ for $g \in G_{2 n}$ and $\psi \in \Psi_{2 n}$. By the choice of $\delta_{2 n}$ and $\Psi_{2 n}$, we can find $w_{2 n+2} \in U(M)$ such that

$$
\left\|u_{g}^{2 n+2} \alpha_{g}^{(2 n)}\left(w_{2 n+2}^{*}\right) w_{2 n+2}-1\right\|_{\phi}^{\#}<\frac{1}{4^{2 n+2}}, \quad g \in G_{2 n}, \phi \in \Phi_{2 n}^{+}
$$

and

$$
(4.2 n+2) \quad\left\|\left[w_{2 n+2}, \varphi\right]\right\|<\frac{1}{4^{2 n+2}}, \quad \varphi \in \Phi_{2 n+1}^{\prime} .
$$

Set $v_{g}^{2 n+2}:=u_{g}^{2 n+2} \alpha_{g}^{(2 n)}\left(w_{2 n+2}^{*}\right) w_{2 n+2}$ and $\alpha_{g}^{(2 n+2)}:=\operatorname{Ad} u_{g}^{2 n+2} \alpha_{g}^{(2 n)}=$ $\operatorname{Ad} v_{g}^{2 n+2} \circ \operatorname{Ad} w_{2 n+2}^{*} \circ \alpha_{g}^{(2 n)} \circ \operatorname{Ad} w_{2 n+2}$. Then

$$
(3.2 n+2) \quad\left\|v_{g}^{2 n+2}-1\right\|_{\phi}^{\#}<\frac{1}{4^{2 n+2}}, \quad g \in G_{2 n}, \phi \in \Phi_{2 n}^{+},
$$

and by $(a .2 n+2)$ and $(b .2 n+2)$, we get

$$
\begin{aligned}
& (1.2 n+2) \quad\left\|\beta_{g}^{(2 n+1)}(\varphi)-\alpha_{g}^{(2 n+2)}(\varphi)\right\|<\frac{1}{2^{2 n}}, \\
& (2.2 n+2) \quad\left\|\beta_{g}^{(2 n+1)}(\psi)-\alpha_{g}^{(2 n+2)}(\psi)\right\|<\frac{\delta_{2 n+1}}{2}, \\
& g \in G_{2 n+1}, \psi \in \bigcup_{g \in G_{2 n+1}} \beta_{g^{-1}}^{(2 n+1)}\left(\Psi_{2 n+1}\right) .
\end{aligned}
$$


Set $\bar{v}_{g}^{2 n+2}:=v_{g}^{2 n+2} \operatorname{Ad} w_{2 n+2}^{*}\left(\bar{v}_{g}^{2 n}\right)$ and

$$
\begin{aligned}
& \Phi_{2 n+3}^{\prime}:=\Phi_{2 n+3} \cup \operatorname{Ad} w_{2 n+2}^{*} w_{2 n}^{*} \cdots w_{2}^{*}\left(\Phi_{2 n+3}\right) \cup\left\{\bar{v}_{g}^{2 n+2} \varphi_{0}, \varphi_{0} \bar{v}_{g}^{2 n+2}\right\}, \\
& \Phi_{2 n+2}^{+}:=\left\{\operatorname{Ad} \bar{v}_{g}^{2 n+2} \varphi_{0} \mid g \in G_{2 n+2}\right\} .
\end{aligned}
$$

We choose $\delta_{2 n+2}>0, \Psi_{2 n+2} \Subset M_{*}$ for $\alpha_{g}^{(2 n)}, 1 / 4^{2 n+4}, G_{2 n+2}, \Phi_{2 n+2}^{+}$and $\Phi_{2 n+3}^{\prime}$ by Lemma 3.4. Then the $(2 n+2)$-nd step is finished, and thus we complete induction.

Set $\theta_{2 n}:=\operatorname{Ad} w_{2 n}^{*} w_{2 n-2}^{*} \cdots w_{2}^{*}$. Then we have $\alpha_{g}^{(2 n)}=\operatorname{Ad} \bar{v}_{g}^{2 n} \circ \theta_{2 n} \circ \alpha_{g} \circ$ $\theta_{2 n}^{-1}$. We will verify $\left\{\theta_{2 n}\right\}$ converges to some $\theta \in \operatorname{Aut}(M)$. To this end, we will prove that $\left\{\theta_{2 n}(\varphi)\right\}$ and $\left\{\theta_{2 n}^{-1}(\varphi)\right\}$ are Cauchy sequences for $\varphi \in M_{*}$. Suppose $\varphi \in \Phi_{k}$. For any $n$ with $k \leq 2 n+1, \varphi$ and $\theta_{2 n}(\varphi)$ are in $\Phi_{2 n+1}^{\prime}$. By $(4.2 n+2)$, we have

$$
\left\|\theta_{2 n+2}(\varphi)-\theta_{2 n}(\varphi)\right\|=\left\|\left[w_{2 n+2}, \theta_{2 n}(\varphi)\right]\right\|<\frac{1}{4^{2 n+2}}
$$

and

$$
\left\|\theta_{2 n+2}^{-1}(\varphi)-\theta_{2 n}^{-1}(\varphi)\right\|=\left\|w_{2 n+2}^{*} \varphi w_{2 n+2}-\varphi\right\|<\frac{1}{4^{2 n+2}} .
$$

It follows that $\left\{\theta_{2 n}(\varphi)\right\}$ and $\left\{\theta_{2 n}^{-1}(\varphi)\right\}$ are Cauchy sequences for $\varphi \in \Phi$. Then so are $\left\{\theta_{2 n}(\varphi)\right\}$ and $\left\{\theta_{2 n}^{-1}(\varphi)\right\}$ for every $\varphi \in M_{*}$, since $\Phi$ is dense in $M_{*}$. Hence $\left\{\theta_{2 n}\right\}$ converges to some $\theta \in \operatorname{Aut}(M)$.

Next we will verify that $\left\{\bar{v}_{g}^{2 n}\right\}$ is a Cauchy sequence with respect to $\|\cdot\|_{\varphi_{0}}^{\#}$. Since we have

$$
\begin{aligned}
\left\|\bar{v}_{g}^{2 n+2}-\bar{v}_{g}^{2 n}\right\|_{\varphi_{0}}^{\#} \leq \|\left(v_{g}^{2 n+2}-1\right) & \bar{v}_{g}^{2 n}\left\|_{\varphi_{0}}^{\#}+\right\|\left(w_{2 n+2}^{*} \bar{v}_{g}^{2 n} w_{2 n+2}-\bar{v}_{g}^{2 n}\right) \|_{\varphi_{0}}^{\#} \\
& +\left\|\left(v_{g}^{2 n+2}-1\right)\left(w_{2 n+2}^{*} \bar{v}_{g}^{2 n} w_{2 n+2}-\bar{v}_{g}^{2 n}\right)\right\|_{\varphi_{0}}^{\#},
\end{aligned}
$$

we will estimate the above three terms.

Suppose $g \in G_{k}$. Then for any $n$ with $2 n \geq k, \varphi_{0}$, Ad $\bar{v}_{g}^{2 n}\left(\varphi_{0}\right) \in \Phi_{2 n}^{+}$, and hence $\left\|v_{g}^{2 n+2}-1\right\|_{\mathrm{Ad} \bar{v}_{g}^{2 n}\left(\varphi_{0}\right)}^{\#}<1 / 4^{2 n+2}$ and $\left\|v_{g}^{2 n+2}-1\right\|_{\varphi_{0}}^{\#}<1 / 4^{2 n+2}$ hold by $(3.2 n+2)$.

We have

$$
\begin{aligned}
\left\|\left(v_{g}^{2 n+2}-1\right) \bar{v}_{g}^{2 n}\right\|_{\varphi_{0}}^{\# 2} & =\frac{1}{2}\left(\left\|\left(v_{g}^{2 n+2}-1\right) \bar{v}_{g}^{2 n}\right\|_{\varphi_{0}}^{2}+\left\|\bar{v}_{g}^{2 n *}\left(v_{g}^{2 n+2 *}-1\right)\right\|_{\varphi_{0}}^{2}\right) \\
& =\frac{1}{2}\left(\left\|v_{g}^{2 n+2}-1\right\|_{\mathrm{Ad} \bar{v}_{g}^{2 n}\left(\varphi_{0}\right)}^{2 n}+\left\|v_{g}^{2 n+2 *}-1\right\|_{\varphi_{0}}^{2}\right) \\
& \leq\left\|v_{g}^{2 n+2}-1\right\|_{\mathrm{Ad} \bar{v}_{g}^{2 n}\left(\varphi_{0}\right)}^{\# 2}+\left\|v_{g}^{2 n+2}-1\right\|_{\varphi_{0}}^{\# 2} \\
& <\frac{2}{16^{2 n+2}}
\end{aligned}
$$


Hence we get $\left\|\left(v_{g}^{2 n+2}-1\right) \bar{v}_{g}^{2 n}\right\|_{\varphi_{0}}^{\#}<\sqrt{2} / 4^{2 n+2}<1 / 2^{2 n+1}$.

We next estimate $\left\|w_{2 n+2}^{*} \bar{v}_{g}^{2 n} w_{2 n+2}-\bar{v}_{2 n}\right\|_{\varphi_{0}}^{\#}$. Since $\varphi_{0}, \bar{v}_{g}^{2 n} \varphi_{0}, \varphi_{0} \bar{v}_{g}^{2 n} \in \Phi_{2 n+1}^{\prime}$, we have $\left\|\left[w_{2 n+2}, \varphi_{0}\right]\right\|<1 / 4^{2 n+2},\left\|\left[w_{2 n+2}, \bar{v}_{g}^{2 n} \varphi_{0}\right]\right\|<1 / 4^{2 n+2}$ and $\left\|\left[w_{2 n+2}, \varphi_{0} \bar{v}_{g}^{2 n}\right]\right\|<1 / 4^{2 n+2}$ by $(4.2 n+2)$. Then

$$
\begin{aligned}
\|\left(w_{2 n+2}^{*}\right. & \left.\bar{v}_{g}^{2 n} w_{2 n+2}-\bar{v}_{g}^{2 n}\right) \cdot \varphi_{0} \| \\
= & \left\|\left(w_{2 n+2}^{*} \bar{v}_{g}^{2 n}-\bar{v}_{g}^{2 n} w_{2 n+2}^{*}\right) w_{2 n+2} \cdot \varphi_{0}\right\| \\
\leq & \left\|\left(w_{2 n+2}^{*} \bar{v}_{g}^{2 n}-\bar{v}_{g}^{2 n} w_{2 n+2}^{*}\right) \cdot \varphi_{0} \cdot w_{2 n+2}\right\| \\
& \quad+\left\|\left(w_{2 n+2}^{*} \bar{v}_{g}^{2 n}-\bar{v}_{g}^{2 n} w_{2 n+2}^{*}\right) \cdot\left[w_{2 n+2}, \varphi_{0}\right]\right\| \\
\leq & \left\|w_{2 n+2}^{*} \bar{v}_{g}^{2 n} \varphi_{0} w_{2 n+2}-\bar{v}_{g}^{2 n} w_{2 n+2}^{*} \varphi_{0} w_{2 n+2}\right\|+2 / 4^{2 n+2} \\
\leq & \left\|\left[w_{2 n+2}^{*}, \bar{v}_{g}^{2 n} \varphi_{0}\right] w_{2 n+2}\right\|+\left\|\bar{v}_{g}^{2 n} \varphi_{0}-\bar{v}_{g}^{2 n} w_{2 n+2}^{*} \varphi_{0} w_{2 n+2}\right\|+2 / 4^{2 n+2} \\
\leq & 1 / 4^{2 n+2}+\left\|\bar{v}_{g}^{2 n}\left[\varphi_{0}, w_{2 n+2}^{*}\right]\right\|+2 / 4^{2 n+2} \\
\leq & 1 / 4^{2 n+1}
\end{aligned}
$$

holds. Hence

$$
\begin{aligned}
\| w_{2 n+2}^{*} & \bar{v}_{g}^{2 n} w_{2 n+2}-\bar{v}_{g}^{2 n} \|_{\varphi_{0}}^{2} \\
& \leq\left\|w_{2 n+2}^{*} \bar{v}_{g}^{2 n} w_{2 n+2}-\bar{v}_{g}^{2 n}\right\|\left\|\left(w_{2 n+2}^{*} \bar{v}_{g}^{2 n} w_{2 n+2}-\bar{v}_{g}^{2 n}\right) \cdot \varphi_{0}\right\| \\
& \leq 2\left\|\left(w_{2 n+2}^{*} \bar{v}_{g}^{2 n} w_{2 n+2}-\bar{v}_{g}^{2 n}\right) \cdot \varphi_{0}\right\| \\
& \leq \frac{2}{4^{2 n+1}}
\end{aligned}
$$

holds by Lemma 2.1(2).

In a similar way, we can show $\left\|\left(w_{2 n+2}^{*} \bar{v}_{g}^{2 n} w_{2 n+2}-\bar{v}_{g}^{2 n}\right)^{*}\right\|_{\varphi_{0}}^{2} \leq 2 / 4^{2 n+1}$. Hence we get $\left\|w_{2 n+2}^{*} \bar{v}_{g}^{2 n} w_{2 n+2}-\bar{v}_{g}^{2 n}\right\|_{\varphi_{0}}^{\#} \leq \sqrt{2 / 4^{2 n+1}}=\sqrt{2} / 2^{2 n+1}<1 / 2^{2 n}$. lows.

The third term $\left\|\left(v_{g}^{2 n+2}-1\right)\left(w_{2 n+2}^{*} \bar{v}_{g}^{2 n} w_{2 n+2}-\bar{v}_{g}^{2 n}\right)\right\|_{\varphi_{0}}^{\#}$ is estimated as fol-

$$
\begin{aligned}
\left\|\left(v_{g}^{2 n+2}-1\right)\left(w_{2 n+2}^{*} \bar{v}_{g}^{2 n} w_{2 n+2}-\bar{v}_{g}^{2 n}\right)\right\|_{\varphi_{0}}^{\# 2} \\
=\frac{1}{2}\left(\left\|\left(v_{g}^{2 n+2}-1\right)\left(w_{2 n+2}^{*} \bar{v}_{g}^{2 n} w_{2 n+2}-\bar{v}_{g}^{2 n}\right)\right\|_{\varphi_{0}}^{2}\right. \\
\left.\quad \quad\left\|\left(w_{2 n+2}^{*} \bar{v}_{g}^{2 n *} w_{2 n+2}-\bar{v}_{g}^{2 n *}\right)\left(v_{g}^{2 n+2 *}-1\right)\right\|_{\varphi_{0}}^{2}\right) \\
\leq 2\left\|w_{2 n+2}^{*} \bar{v}_{g}^{2 n} w_{2 n+2}-\bar{v}_{g}^{2 n}\right\|_{\varphi_{0}}^{2}+2\left\|v_{g}^{2 n+2 *}-1\right\|_{\varphi_{0}}^{2} \\
\leq 4\left\|w_{2 n+2}^{*} \bar{v}_{g}^{2 n} w_{2 n+2}-\bar{v}_{g}^{2 n}\right\|_{\varphi_{0}}^{\# 2}+4\left\|v_{g}^{2 n+2 *}-1\right\|_{\varphi_{0}}^{\# 2} \\
\leq \frac{4}{4^{2 n}}+\frac{4}{4^{2 n+2}} \\
\leq \frac{2}{4^{2 n-1}} .
\end{aligned}
$$


Hence $\left\|\left(v_{g}^{2 n+2}-1\right)\left(w_{2 n+2}^{*} \bar{v}_{g}^{2 n} w_{2 n+2}-\bar{v}_{g}^{2 n}\right)\right\|_{\varphi_{0}}^{\#} \leq \sqrt{2} / 2^{2 n-1}<1 / 2^{2 n-2}$. Summing up, we have the following.

$$
\left\|\bar{v}_{g}^{2 n+2}-\bar{v}_{g}^{2 n}\right\|_{\varphi_{0}}^{\#} \leq \frac{1}{2^{2 n+1}}+\frac{1}{2^{2 n}}+\frac{1}{2^{2 n-2}} \leq \frac{1}{2^{2 n-3}} .
$$

It follows that $\left\{\bar{v}_{g}^{2 n}\right\}$ is a Cauchy sequence and converges to some unitary $\hat{v}_{g}^{0}$.

In the same way, we can show that $\operatorname{Ad} w_{2 n+1}^{*} w_{2 n-1}^{*} \cdots w_{1}^{*}$ and $\bar{v}_{g}^{2 n+1}$ converge to some $\sigma \in \operatorname{Aut}(M)$ and $\hat{v}_{g}^{1} \in U(M)$ respectively. By (1.n) we get Ad $\hat{v}_{g}^{0} \circ \theta \circ \alpha_{g} \circ \theta^{-1}=\operatorname{Ad} \hat{v}_{g}^{1} \circ \sigma \circ \beta_{g} \circ \sigma^{-1}$, and hence $\alpha$ and $\beta$ are cocycle conjugate. By construction, $\theta$ and $\sigma$ are approximately inner.

We will choose a cocycle close to 1 . Suppose $\operatorname{Ad} v_{g} \alpha=\theta \circ \beta_{g} \circ \theta^{-1}$, $\theta \in \overline{\operatorname{Int}}(M)$. Fix $F \Subset G$ and $\varepsilon>0$. Then there exists a unitary $w$ such that $\left\|w v_{g} \alpha_{g}\left(w^{*}\right)-1\right\|_{\varphi_{0}}^{\#}<\varepsilon$ for each $g \in F$. (See the remark after Lemma 3.4.) Define a new $\alpha$-cocycle $v_{g}^{\prime}$ by $v_{g}^{\prime}:=w v_{g} \alpha_{g}\left(w^{*}\right)$. We then have $\left\|v_{g}^{\prime}-1\right\|_{\varphi_{0}}^{\#}<\varepsilon$ for $g \in F$, and

$$
\begin{aligned}
\operatorname{Ad} v_{g}^{\prime} \alpha_{g} & =\operatorname{Ad}\left(w v_{g} \alpha_{g}\left(w^{*}\right)\right) \circ \alpha_{g} \\
& =\operatorname{Ad} w \circ \operatorname{Ad} v_{g} \circ \alpha_{g} \circ \operatorname{Ad} w^{*} \\
& =\operatorname{Ad} w \circ \theta \circ \beta_{g} \circ \theta^{-1} \circ \operatorname{Ad} w^{*} .
\end{aligned}
$$

Put $\sigma:=\operatorname{Ad} w \circ \theta$. Then $\sigma \in \overline{\operatorname{Int}}(M)$, and we get $\operatorname{Ad} v_{g}^{\prime} \alpha_{g}=\sigma \circ \beta_{g} \circ \sigma^{-1}$.

We present applications of Theorem 3.1. Let $M$ be an injective factor. By the Connes-Krieger-Haagerup classification of injective factors [2], [12], [5], [7], $M$ is a McDuff factor. Since $\operatorname{Ker}(\bmod )=\overline{\operatorname{Int}}(M)$ by [3] and [11], we get the following corollary.

Corollary 3.5. Let $M$ be an injective factor, $G$ a discrete amenable group, and $\alpha, \beta$ centrally free actions of $G$ on $M$. Then $\operatorname{Ad} v_{g} \alpha_{g}=\theta \circ \beta_{g} \circ \theta^{-1}$ for some $\alpha$-cocycle $v_{g}$ and $\theta \in \overline{\operatorname{Int}}(M)$ if and only if $\bmod (\alpha)=\bmod (\beta)$. (In the type $I I_{1}$ case, we regard $\bmod (\alpha)$ as trivial for $\alpha \in \operatorname{Aut}(M)$.)

Theorem 3.1 can be modified for a relative McDuff subfactor $N \subset M$ by appropriate changes. Indeed in the proof we only have to replace $M_{\omega}$ with $M_{\omega} \cap N^{\omega}$, which is a subfactor version of a central sequence algebra. Especially if $N \subset M$ is a strongly amenable subfactor of type $\mathrm{II}_{1}$ in the sense of Popa [18], then it is relatively McDuff thanks to Popa's classification theorem for strongly amenable subfactors of type $\mathrm{II}_{1}$ [18]. We also have $\overline{\operatorname{Int}}(M, N)=\operatorname{Ker} \Phi$ by [13], where $\Phi(\alpha)$ is the Loi invariant for $\alpha \in \operatorname{Aut}(M, N)$, and the equivalence between strong outerness and central freeness by [17]. (Also see [14] for the latter fact.) Hence Theorem 3.1 gives an alternative proof of the main theorem in [17]. 
Corollary 3.6. Let $N \subset M$ be a strongly amenable subfactor of type $I I_{1}, G$ a discrete amenable group, and $\alpha, \beta$ strongly outer actions of $G$ on $N \subset M$. Then $\operatorname{Ad} v_{g} \alpha_{g}=\theta \circ \beta_{g} \circ \theta^{-1}$ for some $\alpha$-cocycle $v_{g} \in U(N)$ and $\theta \in \overline{\operatorname{Int}}(M, N)$ if and only if $\Phi(\alpha)=\Phi(\beta)$.

When $N \subset M$ is a strongly amenable subfactor of type $\mathrm{II}_{\infty}$, we have $\overline{\operatorname{Int}}(M, N)=\operatorname{Ker} \Phi \cap \operatorname{Ker}(\bmod )$. Hence we have the following corollary.

Corollary 3.7. Let $N \subset M$ be a strongly amenable subfactor of type $I I_{\infty}, G$ a discrete amenable group, and $\alpha, \beta$ strongly outer actions of $G$ on $N \subset M$. Then $\operatorname{Ad} v_{g} \alpha_{g}=\theta \circ \beta_{g} \circ \theta^{-1}$ for some $\alpha$-cocycle $v_{g} \in U(N)$ and $\theta \in \overline{\operatorname{Int}}(M, N)$ if and only if $\Phi(\alpha)=\Phi(\beta)$ and $\bmod (\alpha)=\bmod (\beta)$.

It is worth noting that Corollary 3.7 yields the classification of strongly amenable subfactor of type $\mathrm{III}_{\lambda}, 0<\lambda<1$. See [13], [17].

\section{REFERENCES}

1. Connes, A., Outer conjugacy classes of automorphisms of factors, Ann. Sci. Eco. Norm. Sup. 8 (1975), 383-420.

2. Connes, A., Classification of injective factors, Ann. of Math. 104 (1976), 73-115.

3. Connes, A., On the classification of von Neumann algebras and their automorphisms, Sympos. Math. XX (1976), 435-478.

4. Connes, A., Periodic automorphisms of the hyperfinite factor of type $I I_{1}$, Acta Sci. Math (Szeged) 39 (1977), 39-66.

5. Connes, A., Type $I I I_{1}$ factors, property $L_{\lambda}^{\prime}$ and closure of inner automorphisms, J. Operator Theory 14 (1985), 189-211.

6. Evans, D. E., and Kishimoto, A., Trace scaling automorphisms of certain stable AF algebras, Hokkaido Math J. 26 (1997), 211-224.

7. Haagerup, U., Connes' bicentralizer problem and uniqueness of the injective factor of type $I I I_{1}$, Acta Math. 158 (1987), 95-148.

8. Izumi, M., Finite group actions on $C^{*}$-algebras with the Rohlin property I, Duke Math. J. 122 (2004), 233-280.

9. Jones, V. F. R., Action of finite groups on the hyperfinite type $I_{1}$ factor, Mem. Amer. Math. Soc 237 (1980).

10. Katayama, Y., Sutherland, C. E., and Takesaki, M., The characteristic square of a factor and the cocycle conjugacy of discrete group actions on factors, Invent. Math. 132 (1998), 331-380.

11. Kawahigashi, Y., Sutherland, C. E., and Takesaki, M., The structure of the automorphism group of an injective factor and the cocycle conjugacy of discrete abelian group actions, Acta Math. 169 (1992), 105-130.

12. Krieger, W., On ergodic flows and the isomorphism of factors, Math. Ann. 223 (1976), 19-70.

13. Loi, P., On automorphisms of subfactors, J. Funct. Anal. 141 (1996), 275-293.

14. Masuda, T., An analogue of Connes-Haagerup approach to classification of subfactors of type $I I I_{1}$, J. Math. Soc. Japan 57 (2005), 959-1003.

15. Nakamura, H., Aperiodic automorphisms of nuclear purely infinite simple $C^{*}$-algebras, Ergodic Theory Dynam. Systems 20 (2000), 1749-1765. 
16. Ocneanu, A., Action of discrete amenable groups on von Neumann algebras, Lecture Notes in Math. 1138 (1985).

17. Popa, S., Classification of actions of discrete amenable groups on subfactors of type II, preprint, 1992.

18. Popa, S., Classification of amenable subfactor of type II, Acta Math. 172 (1994), 163-255.

19. Sutherland, C. E., and Takesaki, M., Actions of discrete amenable groups on injective factors of type $I I I_{\lambda}, \lambda \neq 1$, Pacific. J. Math. 137 (1989), 405-444.

GRADUATE SCHOOL OF MATHEMATICS

KYUSHU UNIVERSITY

FUKUOKA, 812-5250

JAPAN

E-mail: masuda@math.kyushu-u.ac.jp 